\title{
Half Gaussian Kernels Based Shock Filter for Image Deblurring and Regularization
}

\author{
Baptiste Magnier ${ }^{1}$, Huanyu $\mathrm{Xu}^{2}$ and Philippe Montesinos ${ }^{1}$ \\ ${ }^{1}$ LGi2P de l'Ecole des Mines d'Alès, Parc scientifique G. Besse, 30035 Nîmes cedex 1 \\ ${ }^{2}$ School of Computer Science and Technology, Nanjing University of Science and Technology, Nanjing, China \\ \{baptiste.magnier, philippe.montesinos\}@mines-ales.fr, HuanyuXu@gmail.com
}

\begin{abstract}
Keywords: Shock filter, image regularization, deblurring, half Gaussian kernel
Abstract: In this paper, a shock-diffusion model is presented to restore both blurred and noisy image. The proposed approach uses a half smoothing kernel to get the precise edge directions, and use different shock-diffusion strategies for different image regions. Experiment results on real images show that the proposed model can effectively eliminate noise and enhance edges while preserving small objects and corners simultaneously. Compared to other approaches, the proposed method offers both better visual results and qualitative measurements.
\end{abstract}

\section{INTRODUCTION}

Image deblurring (Rosenfeld and Kak, 1982) is a process of removing unwanted blur in the image. As image regularization, it is a crucial image processing step in various applications such as remote sensing, medical image processing, computer vision and so on. They are fields that had largely benefited from techniques of Partial Differential Equations (PDEs). PDEs belong to one of the most important part of mathematical analysis and are closely related to the physical world (Aubert and Kornprobst, 2006). In this context, images are considered as evolving functions of time and a regularized image can be seen as a version of the original image at a special scale. The advantages of using PDEs in image processing arise from their well-established theoretical basis and extensive use in the mathematics, hence allow for a straightforward extension to image processing tasks.

The non-linear diffusion processes have been widely used in the last decade in edge preserving denoising. In order to regularize a grey level image $I: \Omega \rightarrow \mathbb{R},\left(\Omega \subset \mathbb{R}^{2}\right)$ by controlling the diffusion, with the second derivatives in orthogonal directions $(\xi \perp \eta)$, respectively in the edge direction called $\xi$ and in the gradient direction labelled $\eta=\frac{\nabla I}{\|\nabla I\|}$, Perona and Malik (Perona and Malik, 1990) have proposed a model described by the following equation at time $t$ :

$$
\frac{\partial I}{\partial t}=c_{\xi} \cdot I_{\xi \xi}+c_{\eta} \cdot I_{\eta \eta}=c_{\xi} \cdot \frac{\partial^{2} I}{\partial \xi^{2}}+c_{\eta} \cdot \frac{\partial^{2} I}{\partial \eta^{2}}
$$

where $c_{\xi}$ and $c_{\eta}$ are coefficients tuning the diffusion.
When $c_{\xi \xi}=c_{\eta \eta}$, the diffusion is isotropic, blurring important structures in the same way as a convolution with a Gaussian kernel. Choosing a non-increasing function of the gradient magnitude $g(\|\nabla I\|)$ such that:

$$
\left\{\begin{array}{l}
c_{\xi}=g(\|\nabla I\|)=e^{\left(-\frac{\|\nabla I\|}{K}\right)^{2}}, \quad K \in \mathbb{R} \\
c_{\eta}=g(\|\nabla I\|)+\|\nabla I\| \cdot g^{\prime}(\|\nabla I\|),
\end{array}\right.
$$

or with $g(\|\nabla I\|)=\frac{1}{\left(1+(\|\nabla I\| / K)^{2}\right)}$, the diffusion process described in eq. 1 can be interpreted as two directional heat flows with different diffusion intensities depending on the weights $\left(c_{\xi}, c_{\eta}\right)$ in the $\eta$ and $\xi$ directions to preserve discontinuities. This selective smoothing with edge enhancement performs a conditional diffusion: when $\|\nabla I\|$ is small, it turns to a strong smoothing within the homogeneous regions of the image and a weak, selective smoothing across non-homogeneous ones. When $c_{\eta}=0$ in eq. 1, the diffusion scheme behaves like the Mean Curvature Motion (MCM) method: $\frac{\partial I}{\partial t}=I_{\xi \xi}$, preserving well edges (Catté et al., 1992). It consists in performing the diffusion only along the tangential direction $\xi$ or along isophotes (i.e. curves of the image surface of constant intensity). Although the approach of Perona-Malik is able to enhance edges, with highly noisy images, generally, the noise is not totally removed because the diffusion process is inhibited and it may generate a lot of undesired artifacts.

The pioneer work of Perona and Malik on anisotropic diffusion has been one of the most influential paper in the area. In the same framework, the seminal contribution of (Osher and Rudin, 1990) 
on shock filters concerning image deblurring problem uses PDEs to enhance edge of the image. Creating shocks at inflection points, the 2D formulation of the original shock filter can be formulated as:

$$
\frac{\partial I}{\partial t}=-\operatorname{sign}\left(I_{\eta \eta}\right) \cdot I_{\eta}
$$

with $I_{\eta}=\|\nabla I\|$ and where:

$$
\operatorname{sign}(x)=\left\{\begin{array}{cl}
1 & \text { if } I>0 \\
0 & \text { if } I=0 \\
-1 & \text { if } I<0
\end{array}\right.
$$

However, any noise added to the signal creates an infinite number of inflection points, disrupting the process completely. Hence, in (Alvarez and Mazorra, 1994), the authors replaced the edge detector $I_{\eta \eta}$ by its convolution with the Gaussian function $G_{\sigma}$, where $\sigma$ represents the standard deviation of the Gaussian. Thus, the filter becomes more robust against noise:

$$
\frac{\partial I}{\partial t}=-\operatorname{sign}\left(G_{\sigma} * I_{\eta \eta}\right) \cdot I_{\eta} .
$$

In order to achieve a complete image restoration purpose, that is deblurring and denoising, Alvarez and Mazorra try to integrate a denoising component into the existing shock filter deblurring model (Alvarez and Mazorra, 1994). Coupling diffusion ( $I_{\xi \xi}$ term) and shock filter, this approach is modeled as:

$$
\frac{\partial I}{\partial t}=C \cdot I_{\xi \xi}-\operatorname{sign}\left(G_{\sigma} * I_{\eta \eta}\right) \cdot \operatorname{sign}\left(G_{\sigma} * I_{\eta}\right) \cdot I_{\eta}
$$

where $\mathrm{C}$ is a strictly positive constant and $\xi$ is the contour direction, used as a balance between anisotropic diffusion behavior and shock effect. Thus,in addition to create shocks at inflection points, the AlvarezMazorra shock filter model diffuses in the edge direction, eliminating noise.

In (Kornprobst et al., 1997) authors extended the above strategy and proposed a combined diffusionreaction-coupling model, this filter uses:

- a diffusion term according to the $M C M$ scheme,

- a reaction term based on the theory of shock filters (Osher and Rudin, 1990),

- a coupling term that keeps the solution close to the original image.

Although Alvarez-Mazorra and Kornprobst et al. shock filters can eliminate the noise when deblurring, it created homogeneous blobs in flat noisy regions that affect the visual appearance. Moreover, the authors noticed both in (Kornprobst et al., 1997) and (Kornprobst et al., 1997), after a certain number of iterations, corner smoothing is produced.

In (Weickert, 2003), the Coherence-Enhancing Shock Filters (CESF) model was proposed, it is the combination of the Coherence-Enhancing Diffusion (CED) in (Weickert, 1999) model and the shock filter theory (eq. 3). The coherence enhancement effect is achieved by steering the shock filtering along the directions yielded by $J_{\rho}(\nabla I)=G_{\rho} *\left(\nabla I \cdot \nabla I^{T}\right)$, a structure tensor, where $G_{\rho}$ represents a Gaussian kernel of standard deviation $\rho$. Using $\omega$ the normalized eigenvector corresponding to the largest eigenvalue that describes the direction where the contrast change is maximal, the CESF is defined as follows:

$$
\frac{\partial I}{\partial t}=-\operatorname{sign}\left(\left(G_{\sigma} * I\right)_{\omega \omega}\right) \cdot\|\nabla I\|
$$

The CESF model behaves like a contrast enhancing shock filter, it enhances well strip structures like the fingerprint images, however creates artificial lines when dealing with noisy or natural images.

Motivated by quantum mechanics and Schrodinger equation, Gilboa proposed in (Gilboa et al., 2004) a generalized complex shock filter for image deblurring and denoising. Based on a complex diffusion term $\Lambda$ regularizing the noise and indicating inflection points, the imaginary value of the solution controls the smoothing process defined as follows:

$$
\frac{\partial I}{\partial t}=-\frac{2}{\pi} \cdot \arctan \left(a \cdot \operatorname{Im}\left(\frac{I}{\theta}\right)\right) I_{\eta}+\Lambda I_{\eta \eta}+\tilde{\Lambda} I_{\xi \xi}
$$

where $(a, \tilde{\Lambda})$ are real constants and $\theta$ is close to zero. Nevertheless this method brings a weak edges enhancement because this filter operates as a diffusion process for a small time whereas shock terms are created for a large time which can blur some edges.

In (Fu et al., 2006), the authors have developed a region-based shock-diffusion scheme. Using a Guassian kernel, the authors divide the image into threetype regions by its smoothed gradient magnitude. For high gradients (such as boundaries of different objects), a shock-type backward diffusion is performed in the gradient direction, and incorporating a forward diffusion in the isophote lines. For medium gradients (such as textures and details), a soft shock-type backward diffusion is performed. Concerning small gradients (such as smoother segments inside different areas or flat regions), an isotropic diffusion is applied:

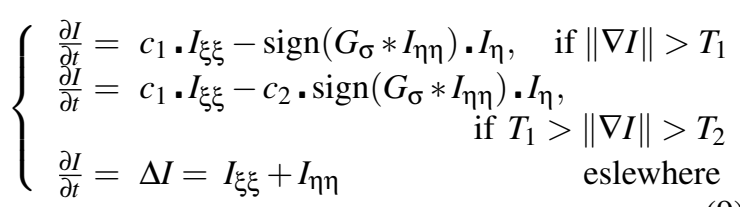

with $c_{1}=\frac{1}{1+\zeta_{1} \cdot I_{\xi \xi}^{2}}$ and $c_{2}=\left|\operatorname{th}\left(\zeta_{2} \cdot I_{\eta \eta}\right)\right|$. The parameters are chosen according to different image regions, $\left(\zeta_{1}, \zeta_{2}\right)$ are constants, and $\left(T_{1}, T_{2}\right)$ are two 
gradient thresholds. Different from a sigmoid function, the hyperbolic tangent function $\operatorname{th}(x)$ guarantees a gradual smoothing transition in areas having medium gradient $\left(T_{1}>\|\nabla I\|>T_{2}\right)$. Note that the $\frac{1}{1+l_{1} \cdot I_{\xi \xi}^{2}}$ term enables to control the diffusion at high curvature edges (Harris and Stephens, 1988), while preserving corners. This shock filter is able to eliminate the noise successfully, but at sharp edges of the restored image is too strong to preserve the original information.

In this paper, we propose a new PDE that combines shock filter with an edge detector using a half Gaussian kernel. The contour detection step brings a more precise direction of the gradient than shock filters using isotropic Gaussian kernels, thus it preserves better corners and small objects of the image. Moreover, the model can solve both deblurring and denoising with both diffusion and the shock filter term.

\section{A Gradient Extraction and Two Edge Directions Estimation}

Steerable isotropic filters (Freeman and Adelson, 1991; Jacob and Unser, 2004) or anisotropic edge detectors (Perona, 1992) perform well in detecting large linear structures (represented in Fig. 1(a) and (b)). Close to corners however, the gradient magnitude decreases as the edge information under the scope of the filter decreases. Consequently, the robustness to noise concerning small objects becomes inappropriate.

A simple solution to bypass this effect is to consider paths crossing each pixel in several directions as in (Sha' ashua and Ullman, 1988). Wedge steerable filters introduced by Simoncelli and Farid (Simoncelli and Farid, 1996) are composed of asymmetric masks providing orientation of edges in different directions issued from a pixel. Unlike the Gaussian function, which is an optimal solution for the Canny criteria(Canny, 1986), wedge steerable filters have a constant amplitude on almost the whole extent of the mask. The idea developed in (Montesinos and Magnier, 2010) was to split the derivative (and smoothing) anisotropic Gaussian kernel in two parts: a first part

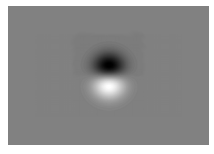

(a) Isotropic gaussian kernel

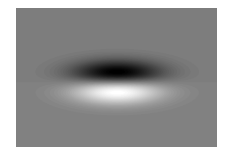

(b) Anisotropic gaussian kernel

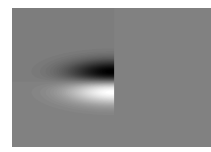

(c) Half anisotropic gaussian kernel
Figure 1: Different 2D derivative Gaussian kernels along an initial direction, and a second part along a second direction (represented in Fig. 4 (a)). At each pixel of coordinates $(x, y)$, a derivation filter is applied to obtain a derivative information $Q(x, y, \theta)$ in function of the orientation $\theta \in[0 ; 2 \pi[$ :

$$
Q(x, y, \theta)=I_{\theta} * C \cdot H(-y) \cdot x \cdot e^{-\left(\frac{x^{2}}{2 \lambda^{2}}+\frac{y^{2}}{2 \mu^{2}}\right)}
$$

where $I_{\theta}$ corresponds to a rotated image ${ }^{1}$ of orientation $\theta, C$ is a normalization coefficient, $(x, y)$ are pixel coordinates, and $(\mu, \lambda)$ the standard deviations of the anisotropic Gaussian filter. Since we only require the causal part of this filter along $Y$ axis, we simply "cut" the smoothing kernel by the middle, in an operation that corresponds to the Heaviside function $H$.

This filter can be compared with isotropic and full anisotropic derivative Gaussian kernels in Fig. 1. $Q(x, y, \theta)$ represents the slope of a line derived from a pixel in the perpendicular direction to $\theta$ (see Fig. 2(b) for several $Q(x, y, \theta)$ signals). We can note that similar filters can also be used for the matching of interest points (Palomares et al., 2012).

To obtain a gradient $\|\nabla I\|$ and its associated direction $\eta$ on each pixel, we first compute with $\theta_{1}$ and $\theta_{2}$ the global extrema of the function $Q(x, y, \theta) . \theta_{1}$ and $\theta_{2}$ define a curve crossing the pixel (an incoming and outgoing direction). Two of these global extrema can then be combined to maximize $\|\nabla I\|$, i.e. :

$$
\left\{\begin{array}{c}
\|\nabla I\|=\underset{\theta \in[0,2 \pi[}{\max } Q(x, y, \theta)-\min _{\theta \in[0,2 \pi[} Q(x, y, \theta) \\
\theta_{1}=\underset{\theta \in[0,2 \pi[}{\arg \max }(Q(x, y, \theta)) \\
\theta_{2}=\underset{\theta \in[0,2 \pi[}{\arg \min }(Q(x, y, \theta))
\end{array}\right.
$$

Fig. 3 shows a gradient image obtained using half Gaussian kernels. Once $\|\nabla I\|, \theta_{1}$ and $\theta_{2}$ have been

\footnotetext{
${ }^{1}$ As explained in (Montesinos and Magnier, 2010), the image is oriented instead of the filter so as to increase algorithmic complexity and moreover allows use of a recursive Gaussian filter (Deriche, 1992).
}

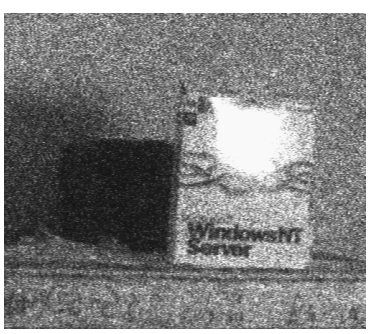

(a) Real noisy image containing high noise $508 \times 440$

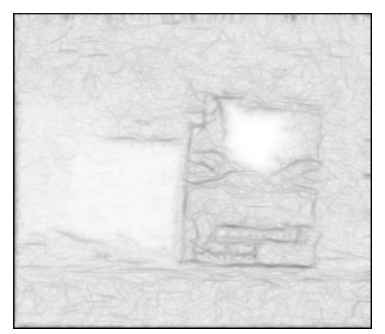

(b) Gradient image
Figure 3: Gradient image (normalized negative image). 


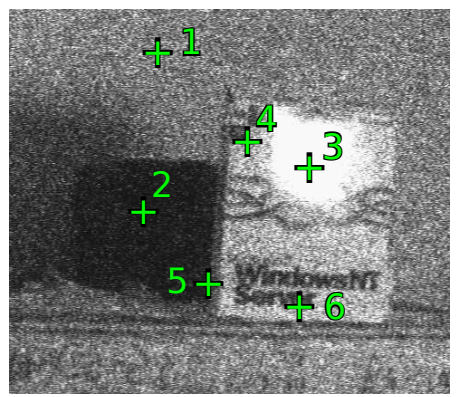

(a) Points selection

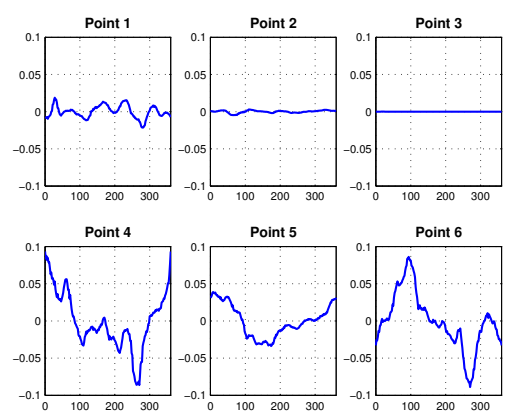

(b) $Q(x, y, \theta)$ for each points of (a)

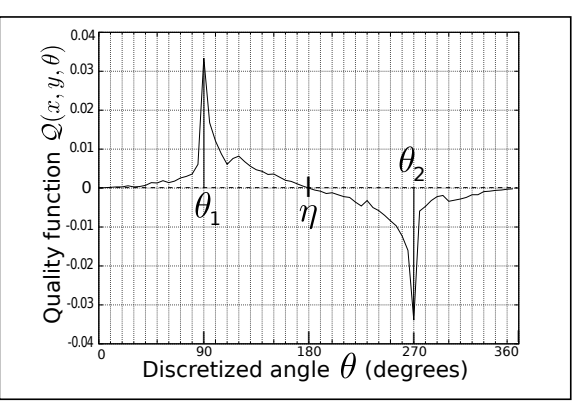

(c) Example of a $Q(x, y, \theta)$ function

Figure 2: Points selection and its associated $Q(x, y, \theta), \mu=10, \lambda=1$ and $\Delta \theta=\frac{\pi}{90}$. Note that the initial orientation of the filter is vertical, upwardly directed and steerable clockwise. In (b), the $X$ axis represents the filter direction in degrees.

obtained, the edges can be easily extracted by computing local maxima of $\|\nabla I\|$ in the direction of the angle $\eta$ (Fig. 2(c) and 4) corresponding to the angle bisector between the two directions $\left(\theta_{1}, \theta_{2}\right)$ :

$$
\eta=\frac{\theta_{1}+\theta_{2}}{2}
$$

Then, a binary image can be built using an hysteresis threshold (see (Montesinos and Magnier, 2010) for further details). In this paper, we are solely interested by the gradient magnitude, the angle formed by the two orientations $\left(\theta_{1}, \theta_{2}\right)$ and the directions $(\eta \perp \xi)$, represented in the diagram in Fig. 4, used in our diffusion scheme discussed below. Moreover, as shown in Fig. 5, half Gaussian kernels enable to extract two precise directions on blurred edges (orientations where the positive and respectively negative slopes are maximum or minimal). Issued from these orientations, diffusion directions $(\eta, \xi)$ are also precise.

Finally, due to their thinness, rotating filters enable computing two precise diffusion orientations in the edge directions, even at high noise levels (Magnier et al., 2012). In (Magnier et al., 2011a), the authors have evaluated the edge detection used in this method with a strong noise level and a comparison with other approaches (Deriche, 1992; Perona, 1992) shows the efficiency of this method.

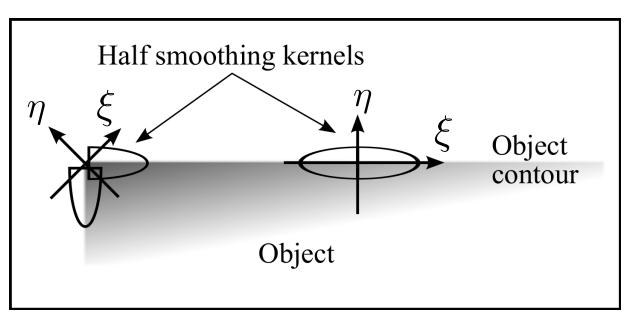

Figure 4: Directions of our diffusion scheme

\section{SIGMOIDS BASED SHOCK FILTER FOR REGIONS}

Images are composed of different regions and features. These regions could be texture or homogeneous image parts. Image enhancing and smoothing are opposite processes, hence, these different parts of the images should be treated differently to obtain the better result. In our shock-diffusion scheme, we divide an image into three-type regions using its gradient magnitude (eq. 11).

Thus, we insert two control functions in our diffusion scheme, which both depend on the gradient magnitude and the angle between the two edge orientations (eq. 11) which is labelled $\beta=\left(\theta_{1}-\theta_{2}\right)$. This $\beta$ angle and the $\eta$ direction are diagramed in Fig. 6 . Concerning high gradients (i.e. greater than a threshold $\tau_{1}$ ), the image is diffused in the tangential direction of edges $\xi$ and a the regularizing process creates a shock in the $\eta$ direction. If the gradient is smaller, in addition to a forward smoothing in the direction $\xi$, a shock-type backward and a forward diffusion are performed in the $\eta$ direction both in function of the gradient level and $\beta$. In the remainder of the image (i.e. low gradient), we apply an isotropic diffusion, smoothing small details as noise in homogeneous regions. Inspired by (Magnier et al., 2012), (Magnier et al., 2011b) and (Fu et al., 2006), involving the gra-
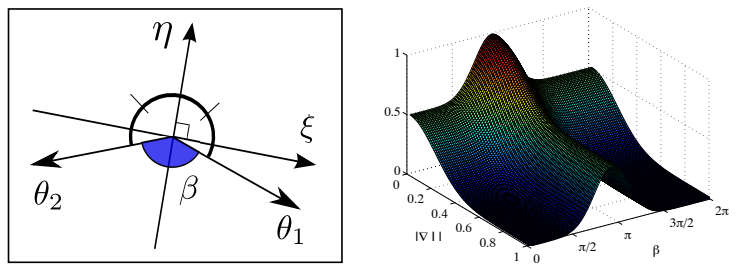

Figure 6: $\beta$ angle, $\eta$ direction, bisector of $\left(\theta_{1}, \theta_{2}\right)$ and the control function $f_{k}$ with $k=0.3$. 

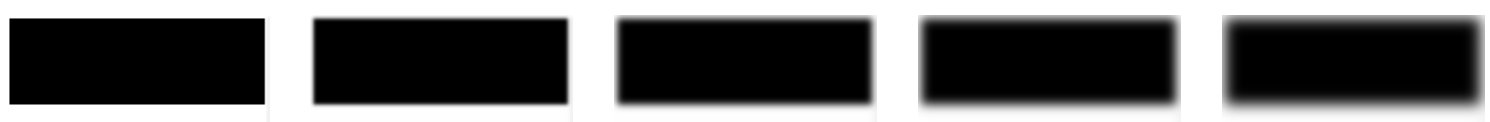

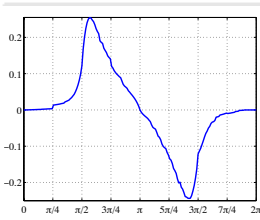

(a) Original image
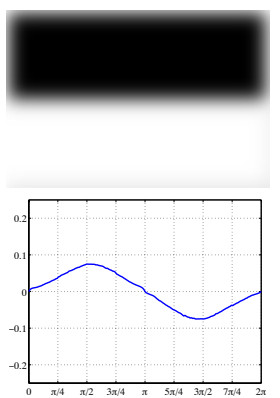

(f) $\sigma=5$

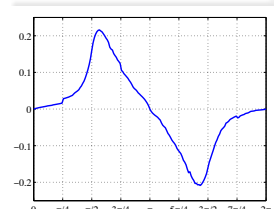

(b) $\sigma=1$
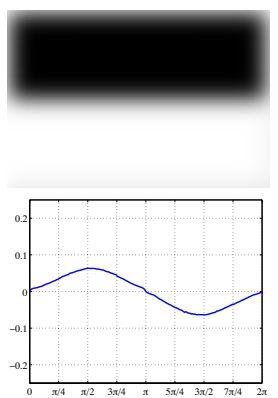

(g) $\sigma=6$

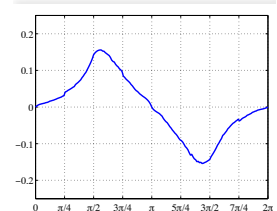

(c) $\sigma=2$
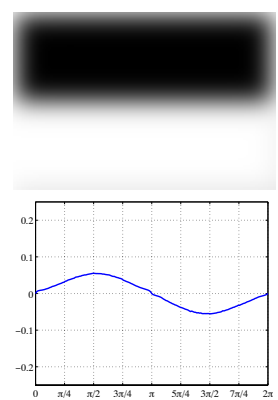

(h) $\sigma=7$

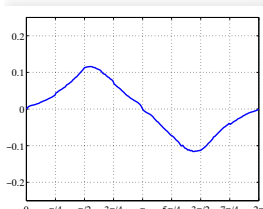

(d) $\sigma=3$
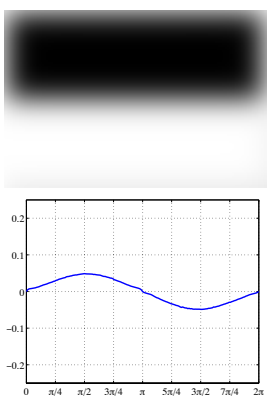

(i) $\sigma=8$

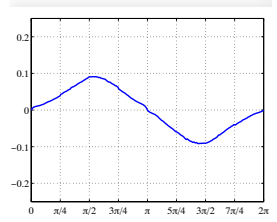

(e) $\sigma=4$

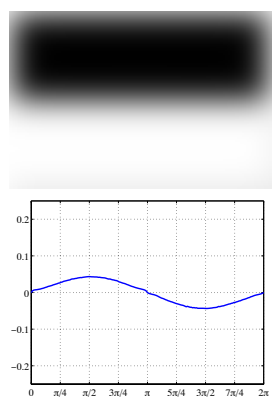

(j) $\sigma=9$

Figure 5: $Q$ signals on a pixel positioned on a step edge in the center of the image in function of the level of a Gaussian blur of standard deviation $\sigma$. The initial orientation of the filter is vertical, upwardly directed and steerable clockwise, with $\lambda=1$, $\mu=5$ and $\Delta \theta=\frac{\pi}{90}$. The maximum of the crests and the minimum of the valleys indicate the orientations of the edges.

dient value and the $\beta$ angle, we present in the following formula our shock-diffusion equation:

$$
\left\{\begin{array}{l}
\frac{\partial I}{\partial t}=f_{k} \cdot I_{\xi \xi}-f_{k} \cdot \operatorname{sign}\left(I_{\eta \eta}\right) \cdot I_{\eta}, \\
\text { for }\|\nabla I\|>\tau_{1} \\
\frac{\partial I}{\partial t}=f_{k} \cdot I_{\xi \xi}+f_{h} \cdot I_{\eta \eta}-f_{k} \cdot \operatorname{sign}\left(I_{\eta \eta}\right) \cdot I_{\eta} \\
\frac{\partial I}{\partial t}=\Delta I=I_{\xi \xi}+I_{\eta \eta} \quad \text { for } \tau_{1}>\|\nabla I\|>\tau_{2} \\
\quad \text { eslewhere }
\end{array}\right.
$$

with $\left(\tau_{1}, \tau_{2}\right)$ two gradient thresholds $\left(\tau_{1}>\tau_{2}\right)$,

$$
\left\{\begin{array}{l}
\left.\left.f_{k}(\|\nabla I\|, \beta)=\frac{e^{-\left(\frac{\|\nabla I\|}{k}\right)}+e^{-\left(\frac{\pi-\beta}{\pi \cdot k}\right)}}{2}, k \in\right] 0,1\right] \\
\left.\left.f_{h}(\|\nabla I\|, \beta)=\frac{e^{-\left(\frac{\|\nabla I\|}{h}\right)}+e^{-\left(\frac{\pi-\beta}{\pi \cdot h}\right)}}{2}, h \in\right] 0,1\right] .
\end{array}\right.
$$

and we impose $k>h$ so that $f_{k}(\|\nabla I\|, \beta)>f_{h}(\|\nabla I\|, \beta)$. In order to ensure a progressive diffusion, $f_{k, h}$ are sigmoids functions, they are represented in Fig. 6.

Note that thresholds $\left(\tau_{1}, \tau_{2}\right)$ are applied only on the gradient magnitude and not a combination with the $\beta$ angle. In fact, a threshold also on the $\beta$ angle would create shocks, resulting in undesirable artifacts in some image parts (e.g. in homogeneous regions).

\section{EXPERIMENTAL RESULTS}

To illustrate the effective of the proposed shock filter with edge detector using a half Gaussian kernels, we present some experimental results . We compare the proposed shock filter with the original one (OR), Alvarez-Mazorra (AM), Gilboa, Weickert (CESF) and Fu et al. (Fu) approaches. Most of the tested images contain blur and noise. In order to measure the objective performance of these models, we compute the PSNR (Peak Signal to Noise Ratio) and the SSIM (Structural SIMilarity presented in (Wang et al., 2004)) before compare each results.

We choose the most suitable parameters for each models. In order to obtain comparative results, we choose the same larger (i.e. standard deviation) of the Gaussian for approaches using this function (i. e. $\sigma=\mu=1)$. For the original shock filter, $d t=$ 0.2 and Alvarez-Mazorra approach, $d t=0.1, C=1$, $\sigma=1$. Parameters used in the Gilboa shock filter are $d t=0.1, \Lambda=0.2, \tilde{\Lambda}=0.4, a=2, \theta=p i / 1000$ and $\sigma=1$. For the CESF model, $\sigma=1, \rho=1$. Concerning algorithm of Fu et al., $d t=0.05, T_{1}=15, T_{2}=5$, $\zeta_{1}=0.0008, \zeta_{2}=300$ and $\sigma=1$. In our method, $d t=0.05, \mu=5, \lambda=1, \Delta \theta=\frac{\pi}{90}$ and $(k, h)$ are changeable in function of the structures of the treated images.

In the two first results, noisy images are produced 


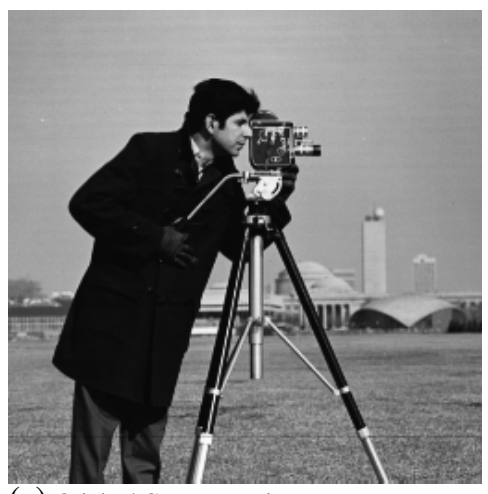

(a) Original Cameraman image $256 \times 256$

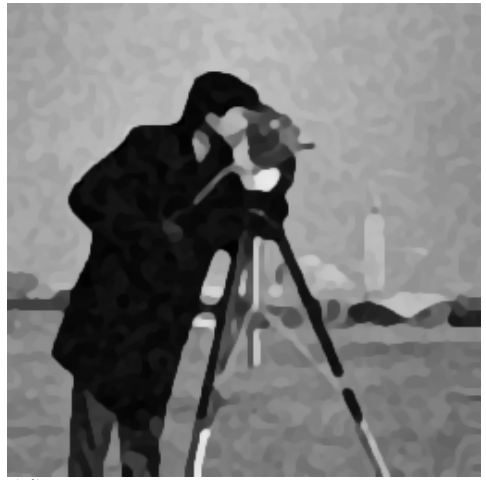

(d) Alvarez-Mazorra shock filter, iteration $=50, \mathrm{PSNR}=22.72, \mathrm{SSIM}=0.715$

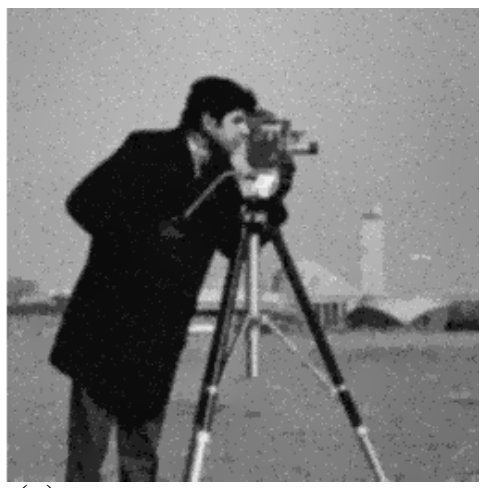

(g) Perona-Malik diffusion, $K=0.02$, iteration $=500$, PSNR $=22.72$, SSIM $=0.715$

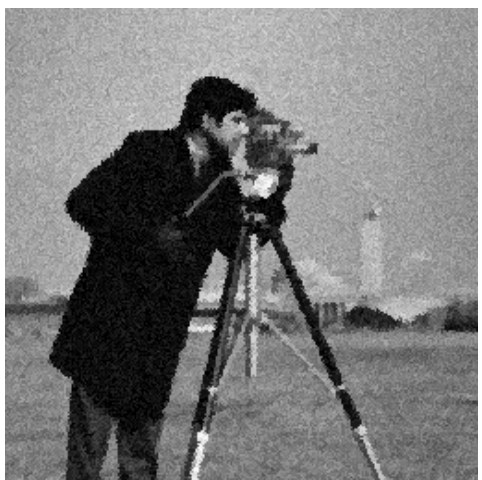

(c) Original shock filter,

iterations $=30$, PSNR $=22.07$, SSIM $=0.420$

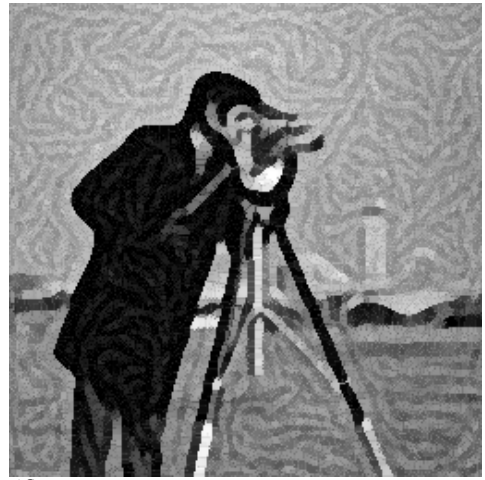

(f) $\mathrm{CESF}$, iteration $=30$, PSNR=19.91, SSIM=0.373

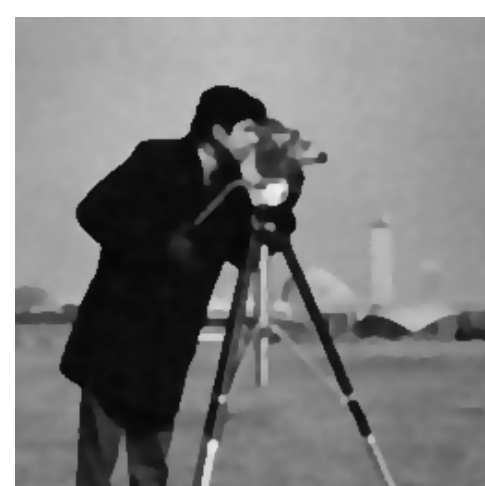

(h) Fu shock filter,

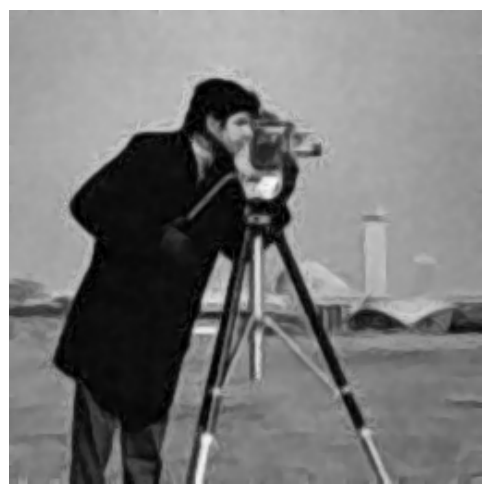

(i) Proposed shock filter,

iteration $=20, \mathrm{PSNR}=25.82, \mathrm{SSIM}=0.792$

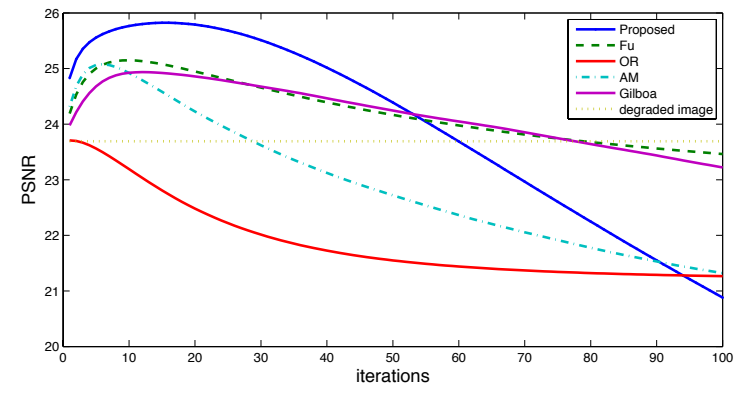

(j) PSNR representation as a function of the number of iterations.

Figure 7: Restoration of Cameraman image by different methods.

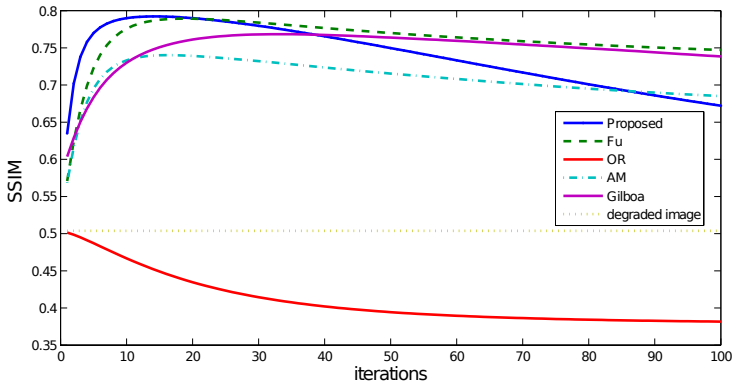

(k) SSIM representation as a function of the number of iterations. 
by adding random Gaussian noise and the blur is caused with the convolution of a Gaussian kernel of standard deviation of $\sigma$.

First, we use a Cameraman image blurred $(\sigma=1)$ and noised $(\sigma=10)$ to compare the performance of the different models (Fig. (7)). The original shock filter and the Perona-Malik method have not very deblurred the image and can not eliminate the noise effectively. AM approach created homogeneous blobs and lost most details. Gilboa filter can smooth the noise, but does not preserve the details of the image (especially in the background). The CESF model performs bad in natural images in noise removal and creates artificial strips. Method of Fu et al. has successfully eliminated the noise, nevertheless, the shock at the edges of the restored image is too strong to preserve the original information so that the result looks like a synthetic image. The proposed model (Fig. 7(i)) eliminates the noise effectively and better enhances the edges than other previous methods as small objects visible in the background. Finally, curves indicate in Figs. 7(j) and (k) that compared to other models, the proposed method can get the highest value both in PSNR and SSIM.

In order to choose better $(k, h)$ parameters of our proposed method, Fig. 8 shows PSNR and SSIM representation of Cameraman image as a function of the iterations number using different values of $(k, h)$. From the curves, we can determine the choice of the ideal game of parameters. For this Cameraman image, blurred and noised, the curves indicate that $k=0.2$ and $h=0.1$ are the best choice for eq. 14 . Moreover, this picture is not so blurred, as the diffusion model is different in function of the two gradient
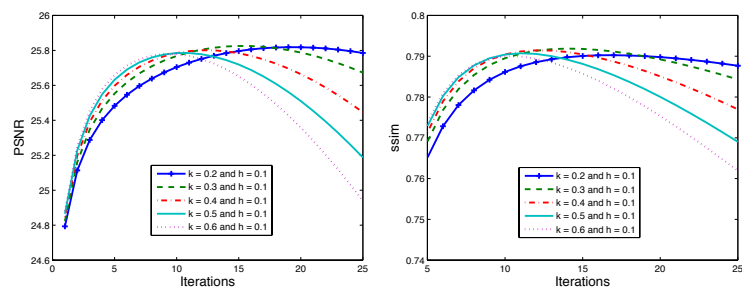

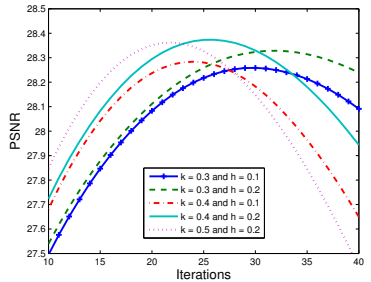

(a) PSNR representation.

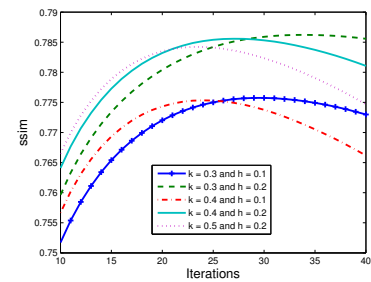

(b) SSIM representation.
Figure 8: PSNR and SSIM representation of Cameraman (top) and House (bottom) images as a function of the iterations number with different games of parameters $(k, h)$. thresholds $\left(\tau_{1}, \tau_{2}\right)$, these two values must not be so high to enhance small objects: $\left(\tau_{1}, \tau_{2}\right)=(0.1,0.15)$.

The next picture in Fig. (9) concerns the House image corrupted by a Gaussian blur $(\sigma=2)$ and a Gaussian noise $(\sigma=10)$. Comparing different methods, the conclusion is the same as the experiments of Cameraman. There are not much texture and small objects in this image, so that methods of Gilboa and Fu et al. can achieve good results. However, the edges of the restored images seem unnatural. Our result has a better visual appearance and small object are better enhanced. Lastly, curves indicate in Figs. 9(j) and (k) that compared to other models, the proposed method can get the highest value both in PSNR and SSIM for this blurred image.

Fig. (8) shows PSNR and SSIM representation of the corrupted House image as a function of the iterations number with different values of $(k, h)$. Curves indicate that $k=0.4$ and $h=0.2$ are the best choice for this image. As this image is more blurred than the Cameraman image, the $k$ value is greater than the previous result. Actually, the more the image is blurred, the more the parameter $k$ in the $f_{k}$ function (eq. 14) must be elevated in order to drive the shock term and diffuse in the $\xi$ direction, enhancing edges. We chose $\left(\tau_{1}, \tau_{2}\right)=(0.5,0.1)$ for this result because this image is more blurred than the Cameraman image and important structures have high normalized gradient.

To verify the effectiveness of the proposed model, we also tested our algorithm on a natural degenerated image (Fig. (10)). Compared to other methods, the proposed approach has the best noise removal result and can preserve the contrast of the original image. Moreover, edges are sharped with our method, it is better visible on the enlargement. These different enlargements of AM and Fu show most homogeneous blobs whereas our results preserve much details while removing efficiently the noise. $\left(\tau_{1}, \tau_{2}\right)=(0.2,0.1)$ for our result with $\tau_{1}$ greater than in the Cameraman image because the considerate image contains a high noise which is not correctly diffused with a lower value of $\tau_{1}$. The choice $(k, h)=(0.3,0.1)$ is done because this image is not so blurred, as the Cameraman.

In order to show the coherence of our algorithm, we apply our diffusion scheme on a fingerprint image. Here, we use $\mu=10, \lambda=1$ to obtain a longer filter such that the algorithm prolongs stripes. We compare our result with the CESF model in Fig. (11). After 300 iterations, our result contains more prolonged filaments and sharped edges show the coherence and the stability of our diffusion scheme. In order to strongly extend lines, $(k, h)=(0.6,0.2)$ and $\left(\tau_{1}, \tau_{2}\right)=(0.1,0.05)$ are relatively low because this image does not contain any noise. 


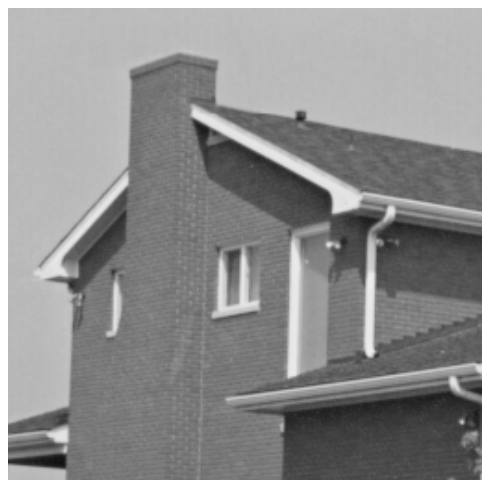

(a) Original House image $256 \times 256$

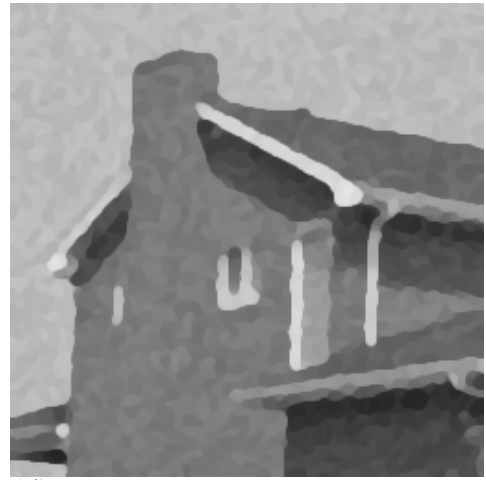

(d) Alvarez-Mazorra shock filter, iteration $=50, \mathrm{PSNR}=25.52, \mathrm{SSIM}=0.734$

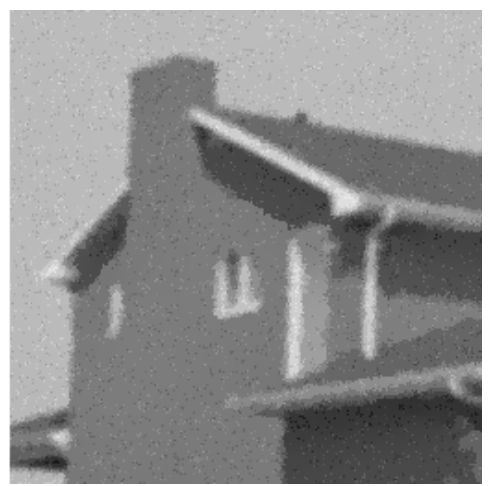

(g) Perona-Malik diffusion, $K=0.02$, iteration $=500, \mathrm{PSNR}=22.72, \mathrm{SSIM}=0.715$

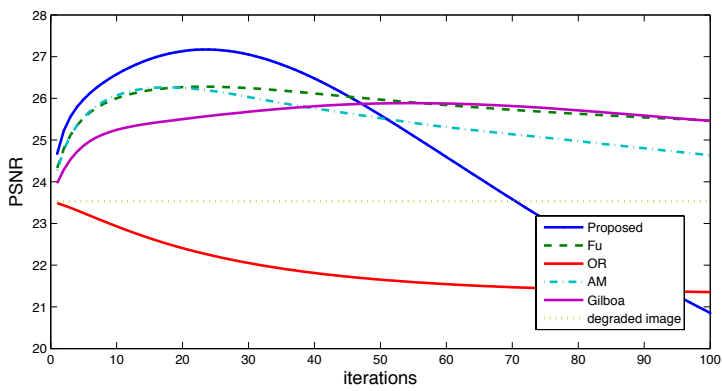

(j) PSNR representation as a function of the number of iterations.

(h) Fu shock filter,

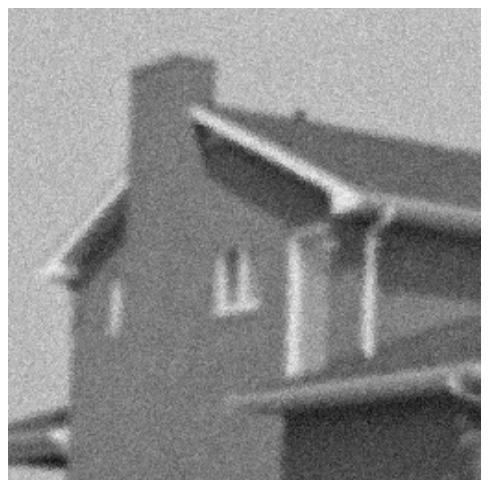

(c) Original shock filter,

(b) Blurred and noised image, PSNR $=23.53$, SSIM $=0.436$

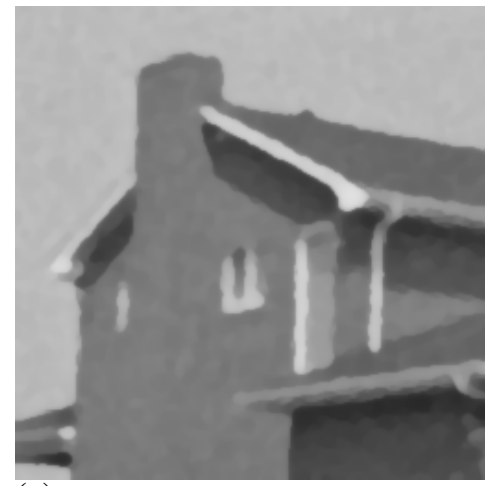

(e) Gilboa complex shock filter, iteration $=30$, PSNR $=25.63$, SSIM $=0.767$

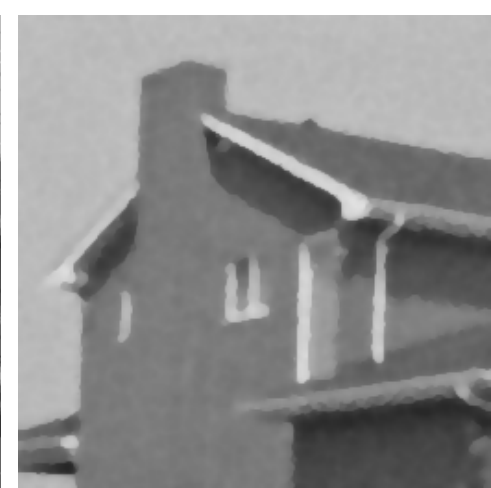
iteration $=30$, PSNR $=22.93$, SSIM $=0.396$

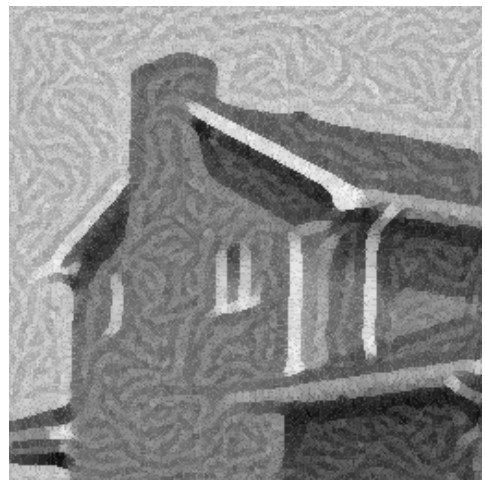

(f) CESF, iteration $=30, \quad$ PSNR $=21.53$ $\mathrm{SSIM}=0.347$

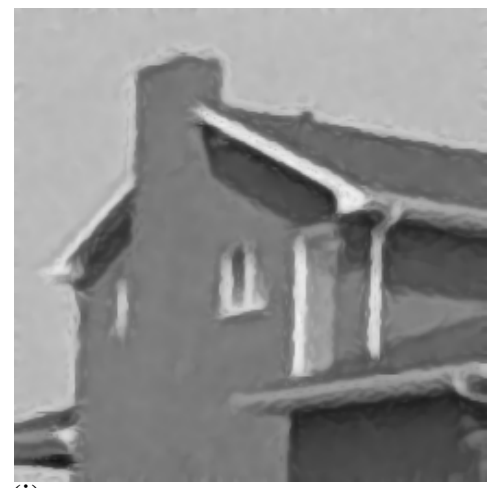

(i) Proposed shock filter,

iteration $=25, \mathrm{PSNR}=26.87, \mathrm{SSIM}=0.781$

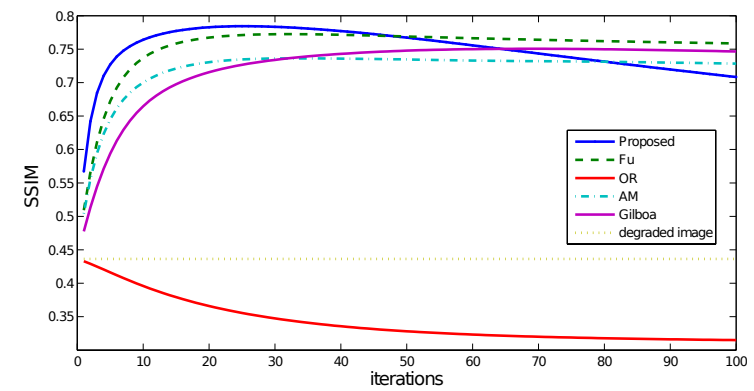

(k) SSIM representation as a function of the number of iterations.

Figure 9: Restoration of House image by different methods. 


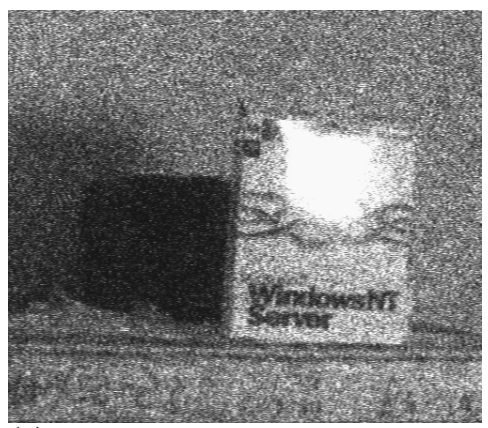

(a) Real degenerated image

$508 \times 445$

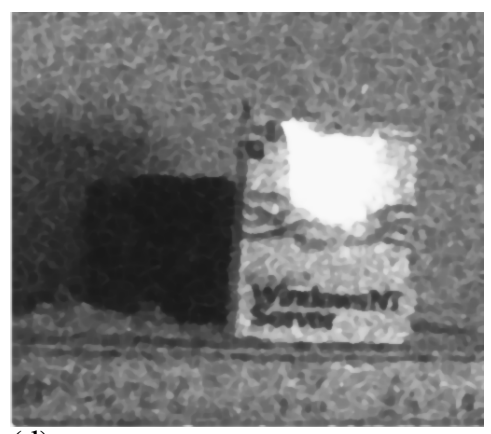

(d) Gilboa complex shock filter,

iteration $=30$

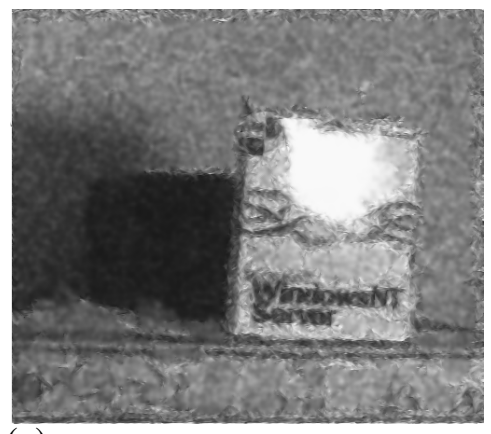

(g) Magnier et al. scheme (Magnier et al., 2012)

iteration $=15$

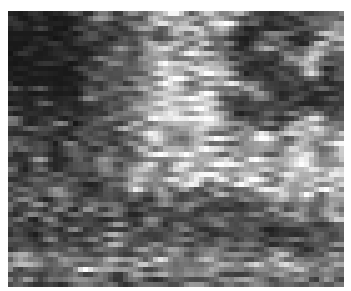

(j) Enlargement of (a)

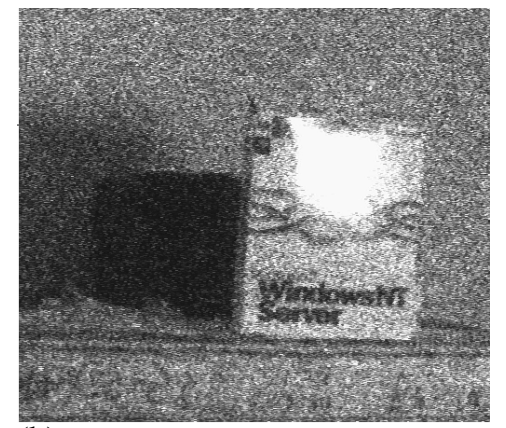

(b) Original shock filter,

iteration $=30$

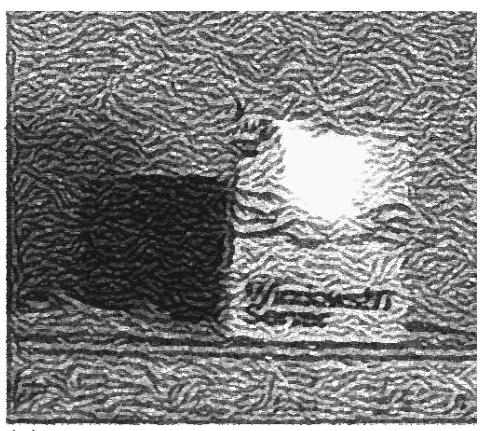

\section{(e) CESF,}

iteration $=30$

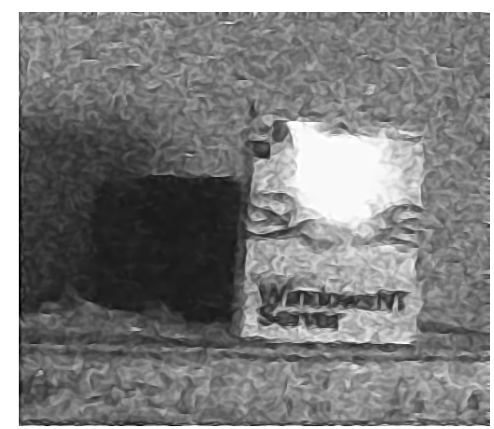

(h) Our result,

iteration $=50$

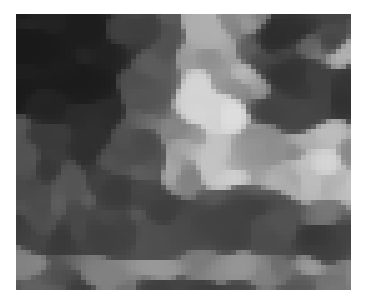

(k) Enlargement of (c)

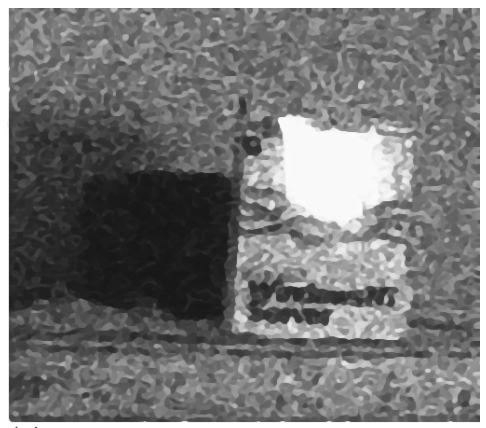

(c) Alvarez-Mazorra shock filter,

iteration $=50$

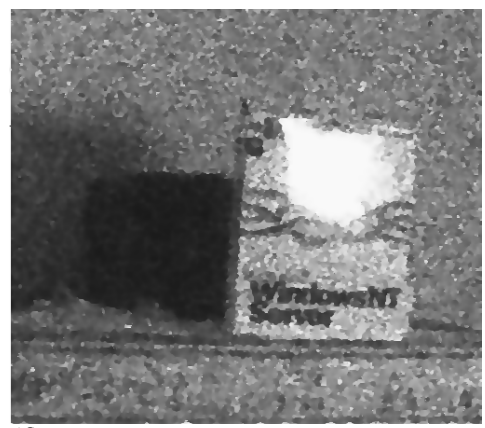

(f) Fu shock filter,

iteration $=30$

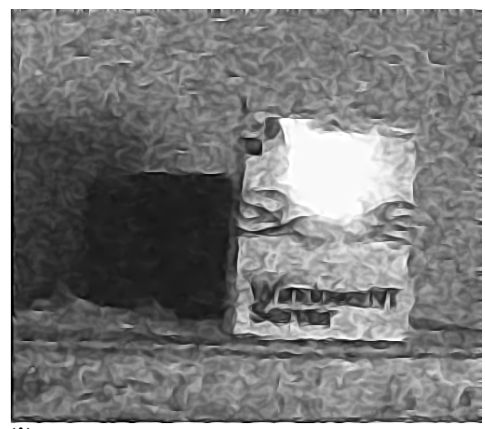

(i) Our result,

iteration $=100$

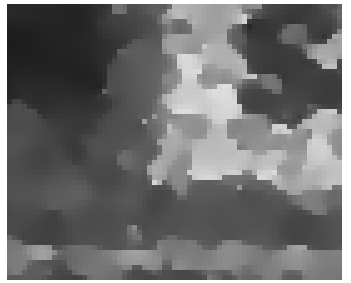

(1) Enlargement of (f)

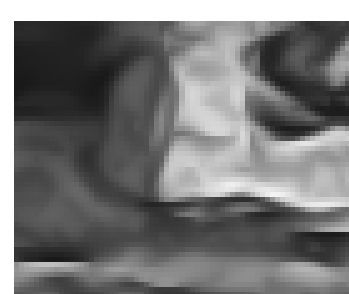

(m) Enlargement of (i)

Figure 10: Restoration of real degenerated image by different methods.

\section{CONCLUSIONS}

In this paper, we have presented a new shockdiffusion filter to restore blurred and noisy image. To make it more efficient, we have introduced new con- trol functions which enable a diffusion process enhancing both edges and corners in the image. The main advantages of our method is that it is based on half Gaussian kernels, extracting precisely the edge directions which enables a preservation of small ob- 


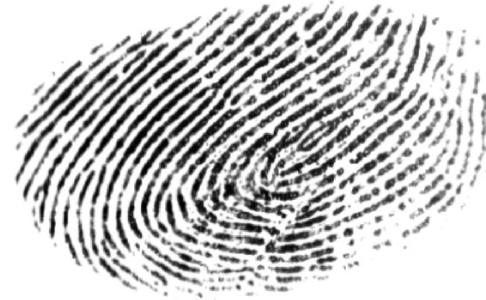

(a) Original fingerprint image, $368 \times 600$

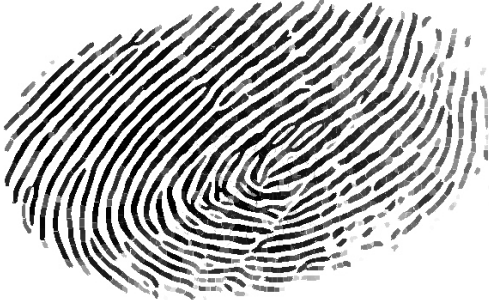

(b) $\mathrm{CESF}$, iteration $=300$

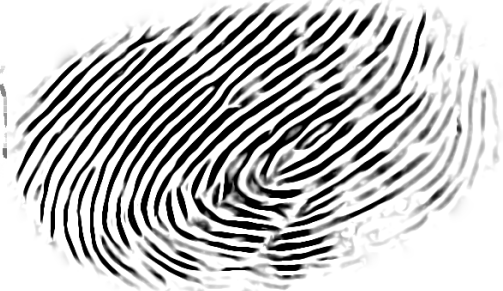

(c) Proposed shock filter, iteration $=300$

Figure 11: Regularization of a fingerprint image.

jects. Finally, the proposed model uses different shock-diffusion strategies on different parts of the image to efficiently eliminate the noise and enhance edges. Experiments on blurred and natural images show that the proposed model can remove noise and sharpen edges effectively, while preserving small objects and corners of the image. As shown in a fingerprint image, this approach is a coherence diffusion method, keeping also the contrast, thus produces better visual quality than the compared models.

\section{REFERENCES}

Alvarez, L. and Mazorra, L. (1994). Signal and image restoration using shock filters and anisotropic diffusion. SIAM J. Numer. Anal., 31(2):590-605.

Aubert, G. and Kornprobst, P. (2006). Mathematical problems in image processing: partial differential equations and the calculus of variations (second edition), volume 147. Springer-Verlag.

Canny, F. (1986). A computational approach to edge detection. IEEE TPAMI, 8(6):679-698.

Catté, F., Lions, P., Morel, J., and Coll, T. (1992). Image selective smoothing and edge detection by nonlinear diffusion. SIAM J. of Num. Anal., pages 182-193.

Deriche, R. (1992). Recursively implementing the gaussian and its derivatives. In ICIP, pages 263-267.

Freeman, W. T. and Adelson, E. H. (1991). The design and use of steerable filters. IEEE TPAMI, 13:891-906.

Fu, S., Ruan, Q., Wang, W., and Chen, J. (2006). Regionbased shock-diffusion equation for adaptive image enhancement. Advances in Machine Vision, Image Processing, and Pattern Analysis, pages 387-395.

Gilboa, G., Sochen, N., and Zeevi, Y. Y. (2004). Image enhancement and denoising by complex diffusion processes. IEEE Trans. Pattern Anal. Mach. Intell., 26(8):1020-1036.

Harris, C. and Stephens, M. (1988). A combined corner and edge detector. In Alvey vision conference, volume 15, page 50. Manchester, UK.

Jacob, M. and Unser, M. (2004). Design of steerable filters for feature detection using canny-like criteria. IEEE TPAMI, 26(8):1007-1019.
Kornprobst, P., Deriche, R., and Aubert, G. (1997). Image coupling, restoration and enhancement via pde's. Image Processing, International Conference on, 2:458.

Magnier, B., Montesinos, P., and Diep, D. (2011a). Fast Anisotropic Edge Detection Using Gamma Correction in Color Images. In IEEE 7th ISPA, pages 212-217.

Magnier, B., Montesinos, P., and Diep, D. (2011b). Texture Removal in Color Images by Anisotropic Diffusion. In VISAPP, pages 40-50.

Magnier, B., Montesinos, P., and Diep, D. (2012). A new region-based pde for perceptual image restoration. In VISAPP, pages 56-65.

Montesinos, P. and Magnier, B. (2010). A New Perceptual Edge Detector in Color Images. In ACIVS, volume 2, pages 209-220.

Osher, S. and Rudin, L. I. (1990). Feature-oriented image enhancement using shock filters. SIAM J. Numer. Anal., 27(4):919-940.

Palomares, J. L., Montesinos, P., and Diep, D. (2012). A New Affine Invariant Method for Image Matching. In IEEE SPIE (3DIP), volume 8290, page 82900Q

Perona, P. (1992). Steerable-scalable kernels for edge detection and junction analysis. IMAVIS, 10(10):663-672.

Perona, P. and Malik, J. (1990). Scale-space and edge detection using anisotropic diffusion. IEEE TPAMI, 12:629-639.

Rosenfeld, A. and Kak, A. C. (1982). Digital Picture Processing. Academic Press, Inc., Orlando, FL, USA, 2nd edition.

Sha' ashua, A. and Ullman, S. (1988). Structural Saliency: The Detection of Globally Salient Structures Using Locally Connected Network. In ICCV, pages 321327.

Simoncelli, E. and Farid, H. (1996). Steerable wedge filters for local orientation analysis. IEEE TIP, 5(9):13771382.

Wang, Z., Bovik, A., Sheikh, H., and Simoncelli, E. (2004). Image quality assessment: From error visibility to structural similarity. IEEE TIP, 13(4):600-612.

Weickert, J. (1999). Coherence-enhancing diffusion filtering. IJCV , 31(2):111-127.

Weickert, J. (2003). Coherence-enhancing shock filters. In Lecture Notes in Computer Science, pages 1-8. Springer. 